\title{
Guest editorial: security requirements engineering: past, present and future
}

\author{
Eric Dubois · Haralambos Mouratidis
}

Published online: 1 January 2010

(C) Springer-Verlag London Limited 2009

\section{Introduction}

Information and software systems become more and more critical in every domain of the human society. Transportation, telecommunications, entertainment, health care, military, education and so on; the list is almost endless. These systems are used not only by major corporations and governments but also across networks of organizations, SME's and by individual users. Such wide use has resulted in these systems containing a large amount of critical information and processes, which inevitably need to remain secure. Therefore, although it is important to ensure that systems are developed according to the user needs, it is equally important to ensure that these systems are secure.

However, the common approach towards the inclusion of security within a software system is to identify security requirements after the definition of a system. This typically means that security enforcement mechanisms have to be fitted into a pre-existing design, leading to serious design challenges that usually translate into the emergence of computer systems afflicted with security vulnerabilities.

Recent research has argued that from the viewpoint of the traditional security paradigm, it should be possible to eliminate such problems through better integration of security and requirements engineering [21]. Security should be considered from the early stages of the

E. Dubois $(\square)$

Centre for IT Innovation, Public Research Centre Henri Tudor, Luxembourg, Luxembourg

e-mail: eric.dubois@tudor.lu

H. Mouratidis

School of Computing, IT and Engineering,

University of East London, London, England

e-mail: haris@uel.ac.uk development process, and security requirements should be defined alongside with the system's requirements specification. Considering security alongside functional requirements helps to limit the cases of security/functional requirements conflict by avoiding them from the very beginning or by isolating them very early in the software system development process.

The need to consider security from the early stages of the software systems development has also been recognized by the requirements engineering community, and relevant publications $[3,7,14,17]$ have been presented that focus on that area. The Journal of Requirements Engineering has been one of the first platforms to discuss and present works that are related to that research area and a guest editorial [3] of the Requirements Engineering Journal on Requirements Engineering for Information Security was published in 2002. This special issue continues that effort.

\section{The past}

At the time of publication, of the 2002 Requirements Engineering for Information Security Special Issue, requirements engineering concerns for the security domain was an emerging area of interest as also demonstrated by various papers published, the same period, at the 2002 IEEE International Requirements Engineering (RE'02) conference [9].

During that time, works considered security requirements from three different perspectives. One perspective [4] was that security requirements are (and should be handled) as another type of non-functional requirements (NFRs). In that direction, specific research was performed to enlarge the spectrum of security requirements and better anchor it in the existing information security domain where 
there was a need for better analysis and specification of security requirements. A second perspective focused on the specification side $[2,12,17,22]$, where publications presented results regarding a better categorization and formalization of security requirements associated to different security problems (like authorization and access systems, high-assurance systems,), and exploiting the link with Common Criteria considered as a reference model for security requirements. A third perspective focused on the elicitation and analysis of security requirements based on a systematic analysis of vulnerabilities and possible attacks, some of them being based on the study of extended use cases [15, 23]. Moreover, an additional research direction was emerging [20], where security requirements were elicited and analysed based on goal-oriented requirements engineering (GORE). Nevertheless, all the different perspectives worked towards one common objective, as indicated in ${ }^{1}$ : To bridge the gap between the information security and requirements engineering communities.

\section{The present}

Since 2002, research has moved towards the satisfaction of two important objectives: On one hand to further reduce the gap between information security and requirements engineering. On the other hand, to investigate security requirements within a broader scope that includes relevant areas of research from the risk management domain. For satisfying these two important objectives, GORE has started to prove its usefulness thanks to the fact that goals vary in their granularity and thus can be used at different decision-making levels.

\subsection{Security requirements}

An important question that has been identified as crucial in order to further reduce the gap between information security and requirements engineering is "what is security requirement?". This question still remains unanswered as the literature fails to provide a widely acceptable definition (to the authors' knowledge). In fact, a large number of research works, published in the literature [14, 17], although they discuss security requirements and provide approaches to analyse them, fail to define them. In other works, security requirements are defined as non-functional requirements, security goals, security solutions, types and levels of protection, security specifications, and security policies. A promising definition for security requirements that seems to receive an increasingly wide acceptance is that security requirements are constraints on system functions $[10,16]$. Our view is consistent with that definition since it allows a unified characterization and understanding of security requirements across the various stages of the development process. Following such characterization of security requirements, the aim for requirements engineers is to elicit appropriate constraints for the system. The challenge is then to define a solution where the system will satisfy its functional requirements without violating its security constraints. Looking at the literature, since the 2002 special issue, we can identify a number of works at different levels that have been developed to define, elicit, analyse security requirements and produce design solutions that satisfy these requirements. In particular, we can identify frameworks, modelling languages, methodologies, patterns and pattern languages. Works vary on the way that the problem is attacked, and the literature [14, 17] provides evidence of researches that are based on UML, on the GORE paradigm, on problem frames and on the idea of patterns. Moreover, each one of these works provides a slightly different perspective on how security requirements are identified, analysed and how security solutions are developed. Taking as an example the Secure Tropos methodology ${ }^{1}[18,19,21]$, which employs a modelling language based on the GORE paradigm, a system is represented as an actor that has a number of goals (strategic interests), plans (ways of achieving their goals) and resources (physical or informational entities that can be used to achieve plans) and might depend on other actors (other systems or stakeholders) for the achievement of some goals. The concept of security constraint is used to model the systems security requirements, and secure entities (secure goals, secure plans, secure resources) are employed to develop a solution in which the system will satisfy its functional requirements without violating its security requirements. In particular, each security constraint is analysed in a number of secure goals, which the actor needs to perform in order to satisfy the security constraint. Such identified secure goals become an actor's security-related strategic interests, and appropriate secure plans and secure resources are put in place to support the satisfaction of the secure goals. The concept of threat is then introduced to model circumstances that have the potential to cause loss or problems and can endanger the security requirements of the system. The concept of attack is also used to analyse the potential threats and identify how best to secure the system's vulnerabilities.

\subsection{Risk management domain}

During the last few years, it has been widely understood that security does not exist in isolation and therefore security requirements should be considered within a

\footnotetext{
${ }^{1}$ We use the Secure Tropos methodology because it is the methodology we are most familiar with.
} 
broader scope, than the boundaries of information security. Towards this direction, work is focused on the integration of security requirements and risk management. The most advanced ideas in the information system security risk management (ISSRM) domain are reported in the new ISO/ IEC $2700 \times$ series regarding information system security management and security risk management. This capitalizes not only on previous standards but also on experience gained and formalized in several security risk management methods (more than 200 methods are registered such as Octave [1], Cramm [6], Ebios [8], and Coras [24]). Moreover, some research work [11-13] has been produced regarding formal definitions of the ontology associated with this domain. The ISSRM domain objective is the protection of essential assets against all harm to information security. As depicted in Fig. 1, assets are things that have value, and can be structured into business assets and Information System (IS) assets. Business assets (information, process, capabilities, employees' skills) are assets related to the organization as components or parts of its business model and are necessary for achieving the associated business strategy. In the health sector, examples of business assets are medical reimbursement processes and patient information. Information System assets are components or parts of the system (e.g., laptop computer, system administrator, network). Note that in many cases, an IS asset (e.g. the patient data record in a database) is the mirror of a business asset (patient information). On the other hand, security criterion is a property or constraint on business assets characterizing their needs for security. It is expressed in terms of confidentiality, integrity and availability (examples of security criteria applied on business assets are confidentiality of the list of patients, integrity of the medical reimbursement process). These security criteria are derived from the business model (strategy) of the organization. A risk is the combination of a threat with one or more vulnerabilities leading to negative impacts harming one or more of the IS assets. Example of a risk in an health information system is an attacker using social

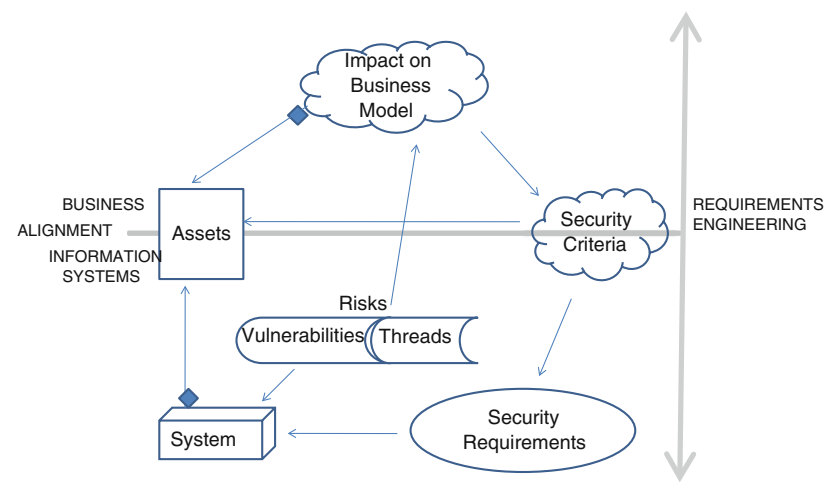

Fig. 1 RE contributions in Business/IT alignment engineering on a member of the organization, because of weak awareness of the staff, leading to non-authorized access on personal computers and loss of confidentiality and integrity of sensitive medical information.

A threat (e.g., an attacker using social engineering) is the potential attack targeting IS assets that may lead to harm. A vulnerability (e.g., weak awareness) is a characteristic of an IS asset that can constitute a weakness or a flaw in terms of security. A threat is composed of a threat agent and an attack method (not depicted in Fig. 1). The impact on the business model is the final consequence of a risk. It is the potential negative consequence of a risk that may harm mostly business assets where it negates security criteria. An impact can provoke a chain reaction of impacts (or indirect impacts), like for example a loss of confidentiality on patient information leads to the non-respect of privacy law and the loss of customer confidence. Finally, the ultimate objective of risk analysis is to provide the adequate information for making the right decision about handling the risk. Depending on factors like e.g. the probability of the risk, the severity of the impact, the cost of the countermeasures, it can be decided to accept the risk or to reduce and/or avoid the risk. In such decision, security requirements are defined to mitigate the risks. In that context, Security requirements are defined as conditions over the phenomena of the environment, which we wish to make true by installing the countermeasure (or control) in the information system (the "machine"), in order to mitigate risks. Note that this definition is effectively the same as the definition we have discussed in the previous section, as it defines security requirements in terms of conditions (constraints).

The ISSRM domain described in the previous paragraph proposes a more holistic view of the security domain. The ISSRM perspective is also fully in line with the challenge of business/IT alignment. On the business side (top part of Fig. 1), the focus of the analysis is on business assets and on their strategic value. On the information system side, the focus is on the mirror of these business assets, i.e. the I.S assets materializing them at the system level. The overall business/IT alignment is guaranteed through the risk analysis component where security criteria associated with business assets are progressively transformed into security requirements and controls materializing them at the implementation level. Here, again we can see the benefits of using GORE for the business part of risk analysis. Highlevel strategic goal models are investigated by several authors in relation with the description of the value proposition included in the business model and associated with its business strategy and the business/regulatory context of the organization. RE approaches proposed at this level provide adequate instruments for reasoning over business assets (assets with a value for the organization) and over 
Impact over Business Objectives (negative consequences on the business model of the organization). Such high-level strategic goal models can be refined in terms of lower-level goals (security criteria) that can be incrementally refined for discovering security requirements.

\section{The future}

As demonstrated by the discussion in the previous two sections, the research area of security requirements engineering is relatively new. Therefore, approaches developed so far are mainly tested on small scale case studies and scenarios. Therefore, more external validation is needed in seeking to demonstrate their effectiveness and usefulness in developing large scale real-life secure software systems. However, the right steps are taken towards this direction, and approaches have been presented lately that demonstrate the applicability and usefulness of some of these approaches in various domains (from banking to health care).

Another interesting direction for future work is the consideration of security within the context of trustworthy software systems. Recent research [5] has argued that security and trust are closely related. This is the case for a number of reasons. For example, security always assumes some degree of trust in its mechanisms. Consider, for instance, a software system that is based on passwords to provide access to an account. The software engineers have assumed that each user is trustworthy and will not make their passwords freely available to potential attackers of the system. Further, the system and its administrator are assumed to be trustworthy not to divulge, alter or remove passwords. Therefore, it is only when such trustworthiness is demonstrated that security can operate properly.

Moreover, as software systems become more and more part of everyday life and personal information is stored, financial transactions take place, and formal and informal communications are supported, the need to understand the implications of relevant laws and regulations to the operation of such systems is becoming an important aspect for software systems developers. From a security requirements engineering perspective, it is important to understand if and how security requirements comply with relevant laws and regulations and how changes in the relevant laws might affect the security requirements of a system. Work in that area has already started and in fact one of the papers in this special issue presents such work. However, a number of important challenges still need to be addressed such as the lack of complete modelling languages to support alignment of security requirements with relevant laws and regulations and procedures and processes to support tracing and reasoning.

\section{Content of this special issue}

The papers presented in this special issue contribute to research directions discussed earlier. To select the five papers presented in this special issue, a thorough review process was followed. The Call For Papers that was distributed for this special issue resulted in 22 submissions, each of which was reviewed by at least three reviewers. The five papers that are presented had to undergo at least two review stages.

The two-first papers illustrate the link, which is progressively established between RE and the ISSRM domain.

- In their paper entitled "A comparison of security requirements engineering methods", Fabian et al. are illustrating how the different reviewed RE methods are covering the different concepts of the ISSRM presented above. The review itself is based on a conceptual framework making explicit the interrelations between the different notions used in security methods.

- While the first paper shows that the different RE security methods are focusing on different parts of the ISSRM domain, the second paper of Elahi et al. proposes a specific notation for modelling and analysing descriptions associated with a whole ISSRM. With the exception of the concept of risk, the paper proposes all the elements of "A Vulnerability-Centric Requirements Engineering Framework" as depicted in Fig. 1.

The three next papers focus on the future challenges as discussed earlier:

- Eliciting security goals (called security objectives in the paper), refine them into security requirements in a systematic way and then trace them to design is addressed in the paper of Islam et al. entitled "Eliciting Security Requirements and Tracing them to Design: An Integration of Common Criteria, Heuristics and UMLsec".

- A more formal approach to the link between security requirements and design is addressed in the paper of Siveroni et al., which presents a framework (USVF) to support the design and verification of secure software systems taking into consideration security and general requirements of the software system.

- At the business strategy level, a negative impact for an organization is the violation of some security and privacy regulations and norms. The paper of Massey et al. proposes a framework for "Evaluating Existing Security and Privacy Requirements for Legal Compliance". Focusing on the healthcare domain, the paper explains and illustrates the challenge of evaluating the compliance of software requirements with legal requirements. 
Acknowledgments We would like to take this opportunity to thank a number of people that contributed to the preparation of this special issue. First of all, we would like to thank all the authors of the submitted papers. We would also like to thank the following for their contribution to the review process: B. De Win, E. B. Fernandez, J. Jurjens, P. Heymans, R. Laleau, A. Mana, N. R. Mead, J. Mylopoulos, G. Sindre, G. Spanoudakis, K. Taguchi, M. Weiss, and E. Yu. Thanks also to Aeronne Jeanne Rivera for the support during the whole preparation process. Last but not least we would like to thank Peri Loucopoulos and Bill Robinson for giving us the opportunity to work on this special issue.

\section{References}

1. Alberts CJ, Dorofee AJ (2001) OCTAVE method implementation guide version 2.0. Carnegie Mellon University, Software Engineering Institute, Pittsburgh, Pennsylvania

2. Anton AI, Earp JB (2004) A requirements taxonomy for reducing web site privacy vulnerabilities. Requir Eng J 9(3):169-185

3. Anton AI (2002) Requirements engineering for information security, guest editorial. Requir Eng J 7(4):177-178

4. Chung L, Nixon B (1995) Dealing with non-functional requirements: three experimental studies of a process-oriented approach, Proceedings of the 17th International Conference on Software Engineering, Seattle-USA, 1995

5. Cofta P (2007) Trust, complexity and control: confidence in a convergent world. Wiley, New Jersey

6. CRAMM website. http://www.cramm.com

7. Devanbu P, Stubblebine S (2000) Software engineering for security: a roadmap, In: Proceedings of the 22nd International Conference on Software Engineering (ICSE '00), Future of Software Engineering Track, pp 227-239, ACM

8. DCSSI (2004) EBIOS-Expression of Needs and Identification of Security Objectives, http://www.ssi.gouv.fr/en/con_dence/ ebiospresentation.html, France

9. Dubois E, Pohl K (eds) (2002) Proceedings of the 10th IEEE Requirements Engineering Conference, Essen, Germany, IEEE Computer Society, ISBN: 0-7695-1465-0

10. Haley C, Laney R, Moffett J, Nuseibeh B (2008) Security requirements engineering: a framework for representation and analysis. IEEE Trans Softw Eng 34(1):133-153

11. Matulevicius R, Mayer N, Mouratidis H, Dubois E, Heymans P, Genon N (2008) Adapting secure tropos for security risk management during early phases of the information systems development. In: Proceedings 20th International Conference on
Advanced Information Systems Engineering (CAiSE'08), Montpellier, France, Lecture Notes in Computer Science, pp 541-555, vol 5074

12. Mayer N (2009) Model-based management of information system security risk. PhD Thesis, University of Namur

13. Mayer N, Heymans P, Matulevicius R (2007) Design of a modelling language for information system security risk management. In: Proceedings of the 1st International Conference on Research Challenges in Information Science (RCIS 2007)

14. McDaniel P, Nuseibeh B (2008) Guest editors' introduction: special section on software engineering for secure systems. IEEE Trans Softw Eng 34(1): 3-4

15. McDermott J, Fox C (1999) Using abuse case models for security requirements analysis. In: Proceedings of the 15th Annual Computer Security Applications Conference (December 06-10, 1999). ACSAC. IEEE Computer Society, Washington, DC, 55

16. Mouratidis H (2004) A security oriented approach in the development of multiagent systems: applied to the management of the health and social care needs of older people in England. $\mathrm{PhD}$ Thesis, University of Sheffield

17. Mouratidis H, Giorgini P (eds) (2006) Integrating security and software engineering: advances and future vision. Idea group, IGI Publishing Groups

18. Mouratidis H, Giorgini P (2007) Secure tropos: a security-oriented extension of the tropos methodology. Int J Softw Eng Knowl Eng (IJSEKE) 17(2):285-309 World Scientific

19. Mouratidis H, Giorgini P (2007) Security attack testing (SAT)testing the security of information systems at design time. Inf Syst 32(8):1166-1183 Elsevier

20. Mouratidis H, Giorgini P, Manson G (2003) Integrating security and systems engineering: towards the modelling of secure information systems. In: Proceedings 15 th International Conference on Advance Information Systems (CAiSE), Velden, Austria, pp 63-78

21. Mouratidis H, Giorgini P, Manson G (2005) When security meets software engineering: a case of modelling secure information systems. Inf Syst 30(8):609-629 Elsevier

22. Schneier B (2000) Secrets \& lies: digital security in a networked world. Wiley, New Jersey

23. Sindre G, Opdahl AL (2000) Eliciting security requirements by misuse cases. In: Proceedings of TOOLS Pacific 2000

24. Vraalsen F, Mahler T, Soldal Lund M, Hogganvik I, den Braber F, Stølen K (2007) Assessing enterprise risk level: the CORAS approach. In: Khadraoui D, Herrmann F (eds) Advances in enterprise information technology security, pp 311-333, Idea group 